\title{
GSK-3 Activity Is Critical for the Orientation of the Cortical Microtubules and the Dorsoventral Axis Determination in Zebrafish Embryos
}

\author{
Ming Shao, Yushuang Lin, Zhongzhen Liu, Ying Zhang, Lifeng Wang, Changbin Liu, Hongwei Zhang* \\ Key Laboratory of Experimental Teratology of the Ministry of Education, Key Laboratory of Animal Cells and Developmental Biology of Shandong Province, Life Science \\ College, Shandong University, Jinan, China
}

\begin{abstract}
The formation of dorsal-ventral $(D-V)$ axis is the earliest event that breaks the radial symmetry and determines the bilateral body plan of a vertebrate embryo, however, the maternal control of this process is not fully understood. Here, we discovered a new dorsalizing window of acute lithium treatment, which covers only less than 10 minutes after fertilization. Lithium treatment in this window was not able to reverse the ventralized phenotype in tokkeabi (tkk) mutant embryos, and its dorsalizing activity on wild-type embryos was inhibited by nocodazole co-treatment. These evidences indicate that the underlying mechanism is independent of a direct activation of Wnt/ $\beta$-catenin signaling, but depends on the upstream level of the microtubule mediated dorsal determinant transport. In order to identify the target of lithium in this newly discovered sensitive window, GSK-3 inhibitor IX as well as the IMPase inhibitor L690, 330 treatments were performed. We found that only GSK-3 inhibitor IX treatment mimicked the lithium treatment in the dorsalizing activity. Further study showed that the parallel pattern of cortical microtubules in the vegetal pole region and the directed migration of the Wnt8a mRNA were randomized by either lithium or GSK-3 inhibitor IX treatment. These results thus revealed an early and critical role of GSK-3 activity that regulates the orientation of the cortical microtubules and the directed transport of the dorsal determinants in zebrafish embryos.
\end{abstract}

Citation: Shao M, Lin Y, Liu Z, Zhang Y, Wang L, et al. (2012) GSK-3 Activity Is Critical for the Orientation of the Cortical Microtubules and the Dorsoventral Axis Determination in Zebrafish Embryos. PLoS ONE 7(5): e36655. doi:10.1371/journal.pone.0036655

Editor: Ramani Ramchandran, Medical College of Wisconsin, United States of America

Received August 27, 2011; Accepted April 4, 2012; Published May 4, 2012

Copyright: (c) 2012 Shao et al. This is an open-access article distributed under the terms of the Creative Commons Attribution License, which permits unrestricted use, distribution, and reproduction in any medium, provided the original author and source are credited.

Funding: This work was supported by the Independent Innovation Foundation of Shandong University, IIFSDU (11200070614069) and China Postdoctoral Science Foundation (10000070311136), the National Natural Science Foundation of China (30570967, 30671072), and the 973 Major Science Programs (2007CB947100, 2007CB815800) from the Ministry of Science and Technology of China. The funders had no role in study design, data collection and analysis, decision to publish, or preparation of the manuscript.

Competing Interests: The authors have declared that no competing interests exist.

* E-mail: zhw@sdu.edu.cn

\section{Introduction}

Dorsal-ventral axis formation is one of the earliest and vital developmental processes that determine the bilateral body plan of all vertebrate embryos. The dorsal organizer plays an important role in this process, and the molecular mechanisms of its induction have been elucidated before [1-7]. However, the upstream maternal control of the dorsal-ventral axis determination is still poorly understood for the moment. In Xenopus and zebrafish, the dorsal-ventral axis is determined shortly after fertilization. In Xenopus, fertilization triggers a "cortical rotation", during which the egg cortex rotates with respect to the sperm entry point. Some proteins together with small granules and organelles move from the vegetal pole region to the perspective dorsal side by polarizedly aligned parallel microtubule arrays [8-10]. Although cortical rotation was not observed in zebrafish embryos [11], parallel microtubule arrays are also present at the vegetal pole about 20 minutes after fertilization (mpf) [12,13]. Depolymerizing this microtubule arrays by UV, cold or nocodazole treatment leads to absence of the dorsal organizer and a ventralized phenotype $[12,14]$. Vegetal yolk ablation before the first cleavage efficiently causes severely ventralized phenotype $[15,16]$. These studies strongly indicate that some "dorsal determinants" (DDs) exist in the vegetal pole region of the zebrafish zygote. This hypothesis was further evidenced in a recent study, which identified the maternalsupplied Wntoa mRNA as one of these determinants [17]. Wntoa transcripts initially located in the vegetal pole after fertilization and were asymmetrically transported to one side of the yolk cortex in a microtubule dependent manner during the first several cell divisions [17].

The DDs are believed to trigger the Wnt/ $\beta$-catenin signaling and cause the stabilization of $\beta$-catenin in the perspective dorsal region. The accumulated cytosolic $\beta$-catenin was observed to enter dorsal cell nuclei at about 128-cell stage in zebrafish embryos $[18,19]$. The ichabod mutant harbors a mutation significantly reducing the expression level and nuclear localization of zebrafish $\beta$-catenin 2, which leads to the loss of organizer gene expression and severely ventralized phenotype [20,21]. This ventralized phenotype can also be achieved by overexpressing Tobl, which can bind $\beta$-catenin and prevent the formation of $\beta$-catenin/LEF1 complex [22]. Nuclear $\beta$-catenin is missing in ventralized embryos caused by blocking the transport of the DDs, like the case in the tokkeabi $(t k k)$ mutant, and early nocodazole or cold treated embryos $[12,23]$. Activating $\mathrm{Wnt} / \beta$-catenin signaling by overexpressing its components like Wnt3, Wnt8, CA- $\beta$-catenin, GBP, Dishevelled, $\mathrm{dn}-\mathrm{Axin} 1$ or $\mathrm{dn}-\mathrm{GSK} 3 \beta$ results in expansion or ectopic formation 
of the dorsal organizer, and can rescue or reverse the ventralized phenotype in tkk mutant embryos [23]. These studies put Wnt/ $\beta$ catenin downstream of the DDs transport. Although the DDs model was established on solid evidence, the regulation of the DDs transport still needs further study.

Lithium salt, known as an anti-psychotic drug, is widely used to control the pathology of the bipolar disorder. The most accepted targets of lithium ion are GSK-3 and the phosphatidylinositol monophosphatase (IMPase) [24,25]. GSK-3 is a component in Wnt signaling, which is inhibited after the canonical Wnt activation. Lithium can noncompetitively inhibit GSK-3 activity, probably by competing with $\mathrm{Mg}^{2+}$ for binding site in this enzyme [26-28]. Owing to this, lithium treatment can mimic the Wnt/ $\beta$ catenin signaling activation by dephosporylating and stabilizing $\beta$ catenin, the direct substrate of GSK-3. And this is widely accepted to interpret the reason why lithium treatment at late cleavage stage causes dorsalization of vertebrate embryos [28]. As GSK-3 participates other metabolic processes and signaling transductions like insulin/insulin-like growth factor signaling, neurotrophic factor signaling and the phosphorylation of microtubule associated proteins [24], it can also regulate many other processes independent of Wnt signaling. IMPase is a key enzyme mediating inositol recycling in the $\mathrm{IP}_{3}-\mathrm{DAG}-\mathrm{Ca}^{2+}$ signaling. Inhibiting this enzyme by lithium causes inositol depletion and eventual shutdown of the $\mathrm{IP}_{3}$-DAG-Ca ${ }^{2+}$ signaling, which is believed as the main mechanism for lithium's pharmacological effects on bipolar disorder [25].

It has been reported that acute lithium treatment at late cleavage stage can cause dorsalization of the zebrafish embryo via activating $\mathrm{Wnt} / \beta$-catenin signaling. Previous studies only observed one sensitive window of lithium treatment [29]. Here in this study, an earlier sensitive window of lithium treatment was discovered, and this sensitive window is limited in an extremely short period, and lasts for only less than $10 \mathrm{~min}$ after fertilization. Although the target of lithium treatment in this window is still GSK-3, the mechanism is completely different from the 32-cell-stage lithium treatment, and depends on microtubule assembly. Further study revealed that the parallel alignment of the vegetal microtubule arrays in response to fertilization and the polarized migration of Wnt 8 a transcripts were randomized by GSK-3 inhibitors. Thus our study revealed for the first time that Wnt/ $\beta$-catenin independent GSK-3 activity is required to regulate the orientation of microtubule arrays and the dorsal determinants transport, and also provided new insight to the different phases of the maternal control during zebrafish dorsal-ventral axis formation.

\section{Results}

\section{Dorsalizing activity of acute lithium treatment exists in two separate windows}

Stachel et al. reported the dorsalizing activity of lithium treatment on zebrafish embryos and showed only one sensitive window from 32-cell stage to sphere stage, before which existed an unresponsive window with an earliest data obtained at 2-cell stage [29]. Here in this study, another sensitive window (SW1 in Figure 1D) was discovered, which was observed just after fertilization with a very short duration of about 10 minutes or less. The zebrafish embryos were synchronizely collected and were treated with $0.3 \mathrm{M}$ lithium chloride $(\mathrm{LiCl})$ solution for $8 \mathrm{~min}$ at specific developmental stages, and the phenotype was analyzed at 12.5 hours post-fertilization (hpf). The results showed that $85.6 \%$ of the embryos treated just after fertilization exhibited a radially dorsalized phenotype similar to the phenotype caused by 32-cellstage lithium treatment [29]. These embryos were radially symmetric and showed a long elliptical shape at the end of gastrulation. The hypoblast cells streamed upwards from the circumference and accumulated at the animal pole (Figure 1A, C serves as a control). The percentage of these radially dorsalized embryos decreased significantly (lowered from $85.6 \%$ to $11.3 \%$ ) when the lithium treatment was carried out 10 minutes later (Figure 1D). After the first cell division (45 mpf), zebrafish embryos gradually turned more and more sensitive to lithium treatment, with increasing percentage of partially dorsalized plus radially dorsalized embryos and decreasing percentage of the normal ones (Figure 1A, B, C, D). At the 32-cell stage $(\sim 105 \mathrm{mpf})$, the dorsalizing activity of lithium treatment was comparable with that of $0 \mathrm{mpf}$ lithium treatment $(85.2 \%$ of radially dorsalized embryos, $11.4 \%$ of mild dorsalized embryos and $4.4 \%$ of normal). The dorsalizing activity of lithium lasts from 32-cell stage until the late blastula stage (Figure 1D), after which lithium treatment mainly caused anterior head truncation instead of dorsalization (data not shown). According to the curves in Figure 1D, we could define three different windows in zebrafish early development: sensitive window 1 (SW1, $0 \mathrm{mpf}$ to $10 \mathrm{mpf}$ ), unresponsive window 1 (UW1, $10 \mathrm{mpf}$ to 32-cell stage), and sensitive window 2 (SW2, 32-cell stage to midblastula stage).

The osmotic stress of $0.3 \mathrm{M} \mathrm{LiCl}$ solution is about 53 fold higher than egg water. To exclude the possibility that any physical factor is responsible for the dorsalizing activity of lithium treatment, we used $0.3 \mathrm{M} \mathrm{NaCl}$ solution as control. The result showed that 8-minute treatment of $0.3 \mathrm{M} \mathrm{NaCl}$ at either 0 -mpf or 32-cell stage had no effect on zebrafish embryogenesis, while most of the 0-mpf and 32-cell stage lithium treated embryos exhibited radially dorsalized phenotype (Figure $1 \mathrm{E}$ ). These results indicate that the dorsalizing activity of lithium treatment in both windows is not dependent on physical factors like the osmotic stress, but on lithium-ion targeting biochemical processes.

\section{Lithium treatment at $0 \mathrm{mpf}$ causes the overall $\beta$ - catenin nuclear localization and the expansion of organizer gene expression}

The dorsal axis specification of zebrafish embryos is dependent on maternal Wnt signaling. Dorsal determinants (DDs) activate $\mathrm{Wnt} / \beta$-catenin signaling in the prospective dorsal margin and stabilize the $\beta$-catenin protein. The stabilized $\beta$-catenin protein enters the nuclei of the dorsal yolk syncytial layer and the dorsal marginal cells and triggers the expression of downstream target genes like bozozok, goosecoid, squint, etc. [30-32]. Lithium treatment at $32-64$ cell stage has been proved to enlarge the region where $\beta$ catenin enters the nuclei, and accordingly causes the expansion of dorsally expressed genes at the expense of ventral markers [19,29]. To test if 0 -mpf lithium treatment has such an effect, we stained the embryos at blastula stage using a $\beta$-catenin antibody. The result showed that the nuclear $\beta$-catenin appeared in the blastomeres located in all directions of mid-blastula embryos after 0 -mpf lithium treatment (Figure 2B). In contrast, the nuclear $\beta$ catenin can only be observed in the dorsal marginal zone of $\mathrm{NaCl}$ treated embryos (Figure 2A). Next, we tested if the expression of the downstream organizer gene goosecoid (gsc) at 50\% epiboly was altered by 0 -mpf lithium treatment. We found that 0 -mpf lithium treatment was able to expand the gsc expression region. More than half $(53.8 \%, \mathrm{n}=26)$ of the embryos presented a circular expression pattern of this gene (Figure 2D), which is consistent with the radially dorsalized phenotype and the wide spread nuclear $\beta$ catenin. However, when lithium treatment was carried out at the 2 cell stage, no embryo presented the circular gsc expression and most $(92.0 \%, \mathrm{n}=25)$ looked rather normal (Figure 2C and $\mathrm{E}$ ). These results indicated that the 0 -mpf lithium treatment can also 

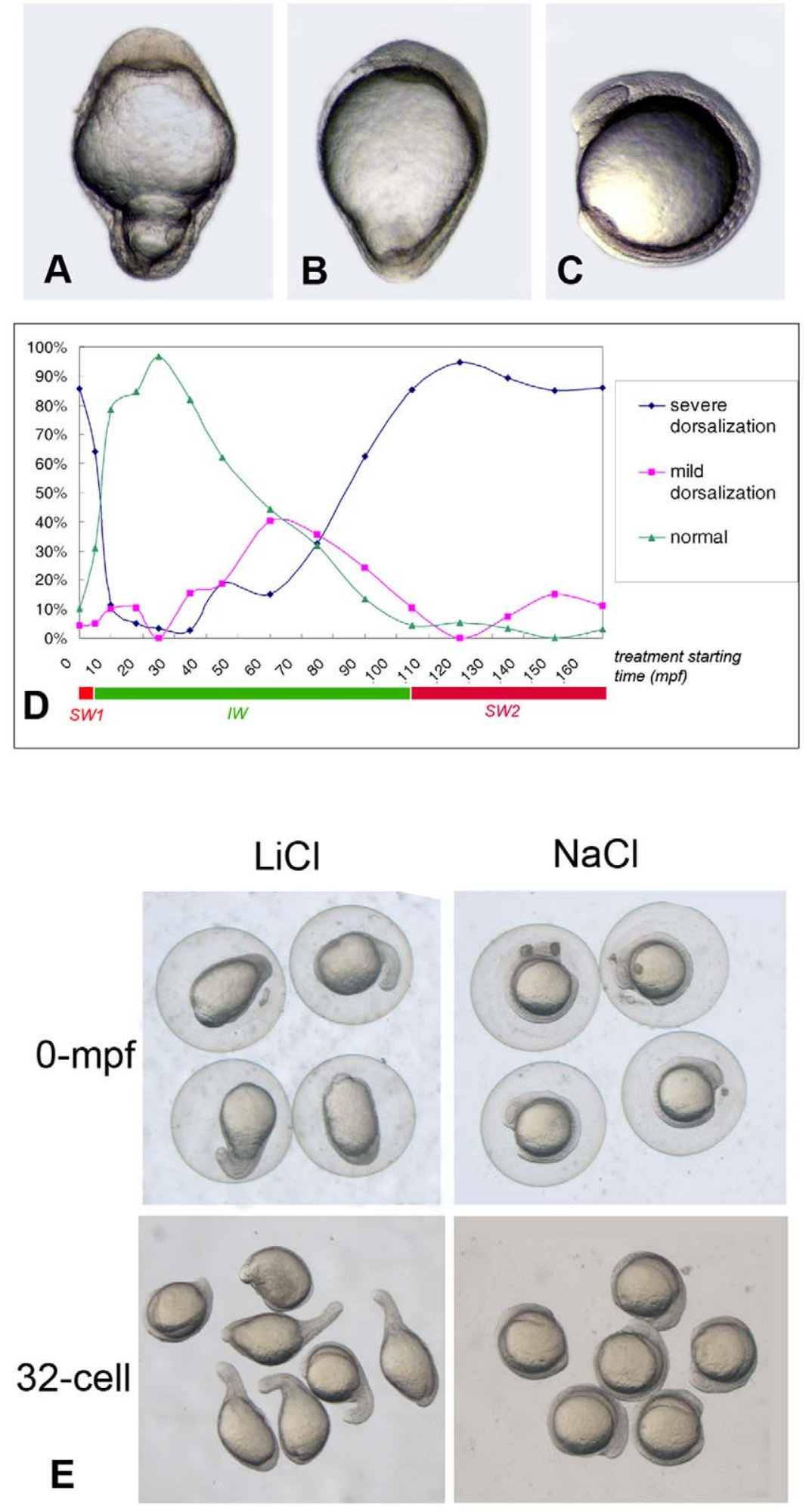

Figure 1. The dorsalizing activity of lithium treatment during zebrafish early development. (A) A severely dorsalized embryo (radialized). (B) A mildly dorsalized embryo. (C) A normal embryo. (D) Diagram demonstrating the dynamics of the dorsalizing capability of acute lithium treatment $(0.3 \mathrm{M} \mathrm{LiCl}$ for $8 \mathrm{~min})$. The abscissa axis designates the time at which lithium treatment began. The ordinate axis designates the percentage of three kinds of embryos with different degrees of dorsalization at $12.5 \mathrm{hpf}$. SW1: Sensitive Window 1; SW2: Sensitive Window 2; UW: Unresponsive Window. The data were obtained in three or more separate experiments, and the number of the embryos used for each data set is more than 100. (E) The dorsalizing effect of the lithium treatment is not caused by osmotic stress by comparing with $\mathrm{NaCl}$ treatment at the same salt concentration and treatment time. Embryos in A, B, C and E was at $12.5 \mathrm{hpf}$, and lateral viewed. The bar in A represents $500 \mu \mathrm{m}$.

doi:10.1371/journal.pone.0036655.g001 


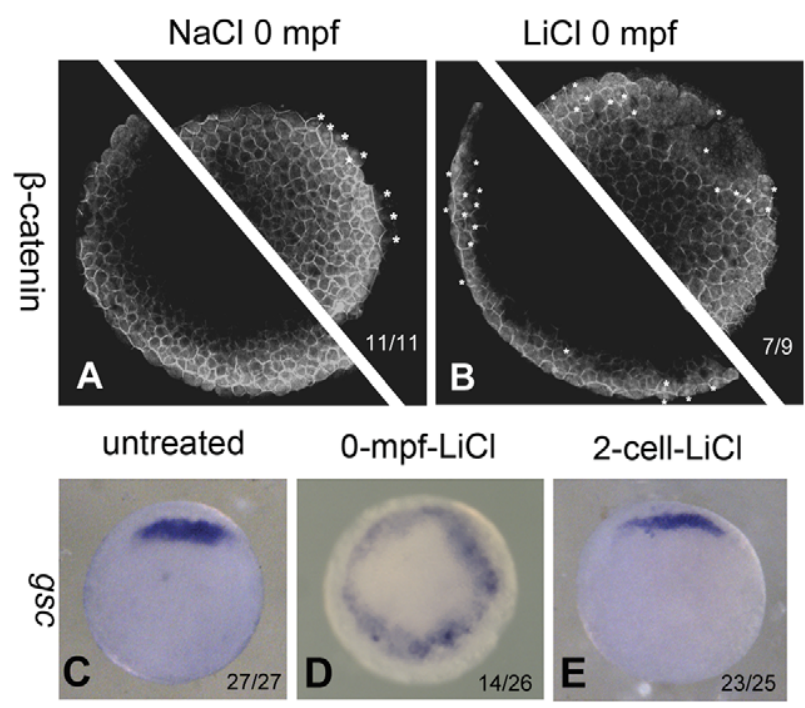

Figure 2. 0-mpf lithium treatment activates Wnt/ $\mathbf{B}$-catenin signaling at mid-blastula stage and expands the organizer region. $(A$ and $B$ ) Confocal immunofluoresence image of of $\beta$-catenin in a 0 -mpf $\mathrm{NaCl}$ treated embryo $(\mathrm{A})$ and a 0 -mpf lithium treated embryo (B) To ensure that the entire marginal zone is investigated, each embryo was scanned for two focal planes near the marginal zone of two hemispheres. The embryos were at sphere stage. Nuclear $\beta$-catenin was marked with white asterisks. (C-E) Expression pattern of organizer gene gsc in untreated (C), 0-mpf lithium treated (D), and 2-cell-stage lithium treated $(\mathrm{E})$ embryos. All the embryos were animal pole view, dorsal up if it can be distinguished.

doi:10.1371/journal.pone.0036655.g002

cause widespread Wnt/ $\beta$-catenin signaling activation in the blastula stage.

\section{The 0-mpf lithium treatment perturbs a microtubule- mediated mechanism upstream of the $\mathrm{Wnt} / \beta$-catenin signaling}

It has been reported that the 32-cell-stage lithium treatment can directly inhibit GSK-3 and stabilize $\beta$-catenin, thus activating the canonical Wnt signaling, and this mechanism is responsible for the dorsalizing activity in the SW2 [28]. The lithium treatment in the SW1 can also cause the expansion of $\beta$-catenin nuclear localization and widespread organizer gene expression. Are the dorsalizing activities of lithium treatment in these two separate sensitive windows via the same mechanism, i.e. by directly activating $\mathrm{Wnt} / \beta$-catenin pathway? To answer this question, we should first find a mutant strain with ventralized phenotype, and the mutant gene should function upstream of the maternal Wnt/ $\beta$ catenin signaling. Tkk is such a maternal mutant in which the function of Kinesin binding protein Syntabulin is lost, so that the transport of the DDs from the vegetal pole to the perspective dorsal region was inhibited, and the embryo exhibits ventralized phenotype [13]. 32-cell-stage lithium treatment can rescue or even reverse the ventralized phenotype of the tkk embryos (personal communication from Dr. Hibi), so if 0-mpf lithium treatment functions via the same mechanism, the ventralized phenotype should also be reversed.

To test this hypothesis, we carried out 0-mpf and 32-cell-stage lithium treatment on both tkk mutant embryos and wild type embryos. The results showed that 0 -mpf lithium treatment cannot rescue or reverse the ventralized phenotype, but in sharp contrast, it synergistically aggravates the ventralized phenotype. Tkk females were crossed with young $\mathrm{AB}$ males, and this cross often generated embryos with low percentage of ventralization. To show the synergistic effect, we used several batches of such embryos with low penetrance from this cross. In these batches, only $7.3 \%$ of embryos presented severely ventralized phenotype (V4), and $13.4 \%$ with moderately ventralized phenotype (V2-V3, Figure 3A, the classification of phenotypes is according to Kishimoto et al. [33], with modifications). When these embryos were subjected to 0 -mpf lithium treatment, the percentage of severe ventralized embryos rose to $17.1 \%$, and the partial ventralized embryos increased to $36.6 \%$. As expected, 32-cellstage lithium treatment can reverse the ventralized phenotype of tkk embryos: no ventralized embryos were observed in this group and more than $64.6 \%$ showed dorsalized phenotype (C2 C5, Figure 3A). To further test this phenomenon, tkk or wild-type (WT) embryos were analysed by in situ hybridization at 50\% epiboly stage using the probe of gsc. As expected, the gsc expression domain was reduced significantly in $t k k$ embryos compared to the wildtype, with $12.0 \%(\mathrm{n}=25)$ no expression. But when 0-mpf lithium treatment was applied to the $t k k$ mutant embryos, the expression of gsc was even much weaker than untreated $t k k$ embryos and the $g s c$ negative embryos rose significantly to $43.5 \%(n=23)$ (Figure 3B, C, D). By measuring the central angle of the gsc expressing crescent, we found that for wild-type embryos, 0-mpf lithium treatment greatly increased the average central angle from $76.7^{\circ}$ to $245.0^{\circ}$. But for $t k k$ mutant embryos, the same lithium treatment caused a significant decrease in the angle (from $36.2^{\circ}$ to $18.3^{\circ}$ ) (Figure $3 \mathrm{E}$ and $\mathrm{F}$ ). These experiments demonstrated that Wnt/ $\beta$ catenin signaling activation cannot explain the dorsalizing activity of 0-mpf lithium exposure. In addition, the results also indicate a possible connectedness between the mechanism of 0 -mpf lithium treatment and Syntabulin associated processes.

\section{Comparison between 0-mpf and 32-cell-stage lithium treatment on dorsal-ventral gene expression}

The dorsalizing activity of 0 -mpf and 32-cell-stage lithium treatment is by way of different mechanisms, which may be reflected by differences in dorsal-ventral gene expression, although no phenotypic differences could be distinguished. To test this possibility, we reexamined by the in situ hybridization 0-mpf and 32-cell-stage lithium treated wild-type and tkk embryos at $50 \%$ epiboly, using gsc and eve1 as dorsal-ventral markers. As expected, several differences were discovered. 0-mpf lithium treatment usually caused a scattered expression of $g s c$, with distinct $g s c$ negative cells in between, and the circular area of $g s c$ expression was much thicker with respect to the untreated or 32-cell-stage lithium treated embryos (Figure 4A, B, C). eve1 expression was reduced, but not absent in 0 -mpf lithium treated embryos, while for 32-cell-stage lithium treated embryos, the expression of eve1 frequently disappeared (Figure 4D, E, F). These results indicated that 0 -mpf lithium treatment is less potent to induce dorsal gene expression or to inhibit ventral gene expression, which is in support of the possibility that 0 -mpf lithium treatment altered the distribution of DDs rather than directly activated the Wnt/ $\beta$ catenin signaling.

For tkk mutant embryos, 0-mpf lithium treatment caused a decrease or disappearance of the $g_{S c}$ expression and the enhancement of eve1 expression (Figure 4G, H, J, K). But the 32-cell-stage lithium treatment can enlarge gsc expression region, and some embryos displayed a circular gsc expression and disappeared eve1 expression (Figure 4I, L), although the frequency was much lower than the treated wild-type embryos. In addition, the width of $g s c$ expression region was significantly thinner than the 32-cell-stage lithium treated wild-type embryos (Figure 4C, I), which is in accordance with the lower percentage of the radially 

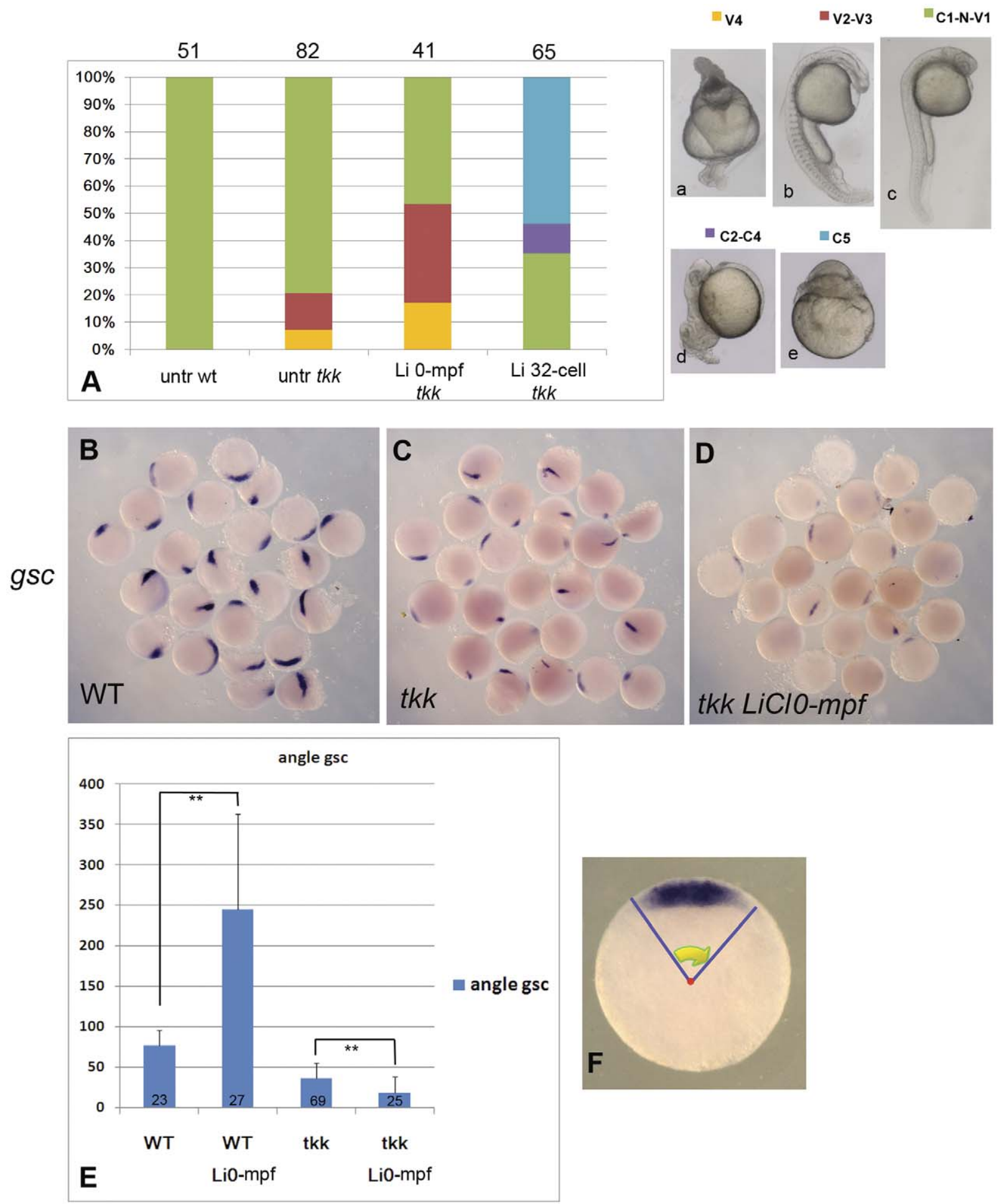

Figure 3. 0-mpf lithium treatment exacerbates the ventralized phenotype of tokkeabi mutant embryos. (A) Phenotypic analysis of lithium treated tkk embryos. We adopted the Dorsoventral Index previously described [33], but some of the categories were combined in order to simplify the statistics, as stated below: (Aa) V4: a representative radially ventralized embryo; (Ab) V2-V3: a moderately ventralized embryo with distinguishable D-V axis but no eyes; (Ac) C1-Normal-V1: embryos with eyes (regardless of the size) and relatively normal D-V axis; (Ad) C2-C4: A partially dorsalized embryo with shortened anterioposterior length; (Ae) C5: A radially dorsalized embryo. (B-D) the expression of gsc in wild-type (B), tkk mutant (C), and 0-mpf lithium treated tkk mutant embryos (D). (E) The central angle of gsc expression showing a significant decrease in 0-mpf lithium treated embryos with respect to wild-type untreated, 0 -mpf lithium treated wild-type and $t k k$ untreated embryos. $(\mathrm{F})$ The measurement of the central angle of $g s c$ expression. The error bars in (E) designate the standard deviation of each data set. ** means that the $p$ value is lower than 0.001 according to the Student's t test. Embryo numbers were designated for each column in (A) and (E).

doi:10.1371/journal.pone.0036655.g003 


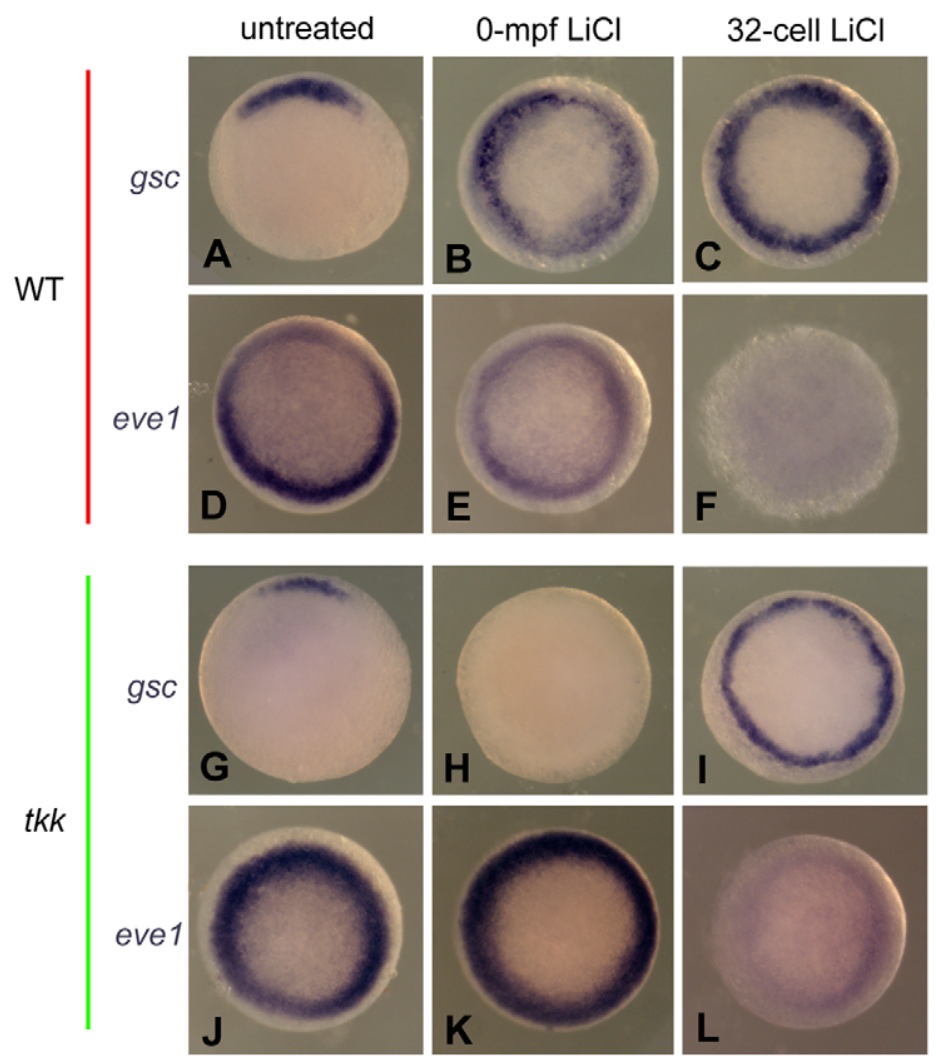

Figure 4. The comparison of dorsal and ventral gene expression between 0-mpf lithium treatment and 32-cell-stage lithium treatment. Representative embryos from indicated groups stained by gsc probe (A-C, and G-I) or eve1 probe (D-F and J-L) at $50 \%$ epiboly. All the embryos are animal pole view and with dorsal side upward if it can be distinguished. doi:10.1371/journal.pone.0036655.g004

dorsalized phenotype for 32-cell-stage lithium-treated tkk mutant embryos. These observations further support that the dorsalizing activities of the 0-mpf and the 32-cell-stage lithium treatment are via different mechanisms. They also suggest that $t k k$ mutant embryos are less sensitive to the 32-cell-stage lithium treatment than the wild-type.

\section{The dorsalizing activity of 0 -mpf lithium treatment is dependent on microtubule assembly}

Vegetal cortical microtubules align parallelly for the directed DDs transport after fertilization. Disrupting the microtubule assembly by nocodazole treatment stopped the polarized migration of Wnt8a mRNA and caused ventralization of zebrafish embryos $[12,17]$. To verify the relationship between 0 -mpf lithium treatment and cortical microtubule assembly, we tested if nocodazole treatment can reverse the dorsalized phenotype caused by the 0 -mpf lithium treatment. The results showed that compared to 0 -mpf $\mathrm{NaCl}$ treated embryos (Figure 5A, E), all the 0 -mpf lithium treated embryos (26/26) showed a typical dorsalized shape with a significantly elongated animal-vegetal axis at $12 \mathrm{hpf}$ (Figure 5C). However, when $0.1 \mu \mathrm{M}$ nocodazole was added to the $0.35 \mathrm{M} \mathrm{LiCl}$ solution and treated at 0 -mpf, all the $12 \mathrm{hpf}$ embryos showed a much round shape, $43.6 \%$ embryos $(n=39)$ with more cells accumulated near the blastopore (Figure 5D), which is similar to the typical ventralized phenotype caused by NaCl-nocodazole co-treatment (27.9\% ventralized, $n=43$ ) (Figure 5B). At $22 \mathrm{hpf}$, the 0-mpf lithium treated embryos showed dorsalized phenotype (radially or with curved or trunked tail) (Figure 5G), while for the LiCl-nocodazole co-treated embryos, ventralized phenotype with no head and enlarged yolk extension dominated the group (Figure $5 \mathrm{H}$ ), very similar to NaCl-nocodazole co-treated embryos (Figure 5F). The changes in the phenotype after adding nocodazole demonstrated that depolymerizing microtubules can block the dorsalizing effect of 0-mpf lithium treatment (statistics shown in Figure 5I), which strongly indicated that the dorsalizing activity of the 0 -mpf lithium treatment requires successful assembly of vegetal cortical microtubules.

\section{The dorsalizing activity of 0 -mpf lithium treatment is not due to the stabilization of microtubules}

Xenopus embryos can be dorsalized by $\mathrm{D}_{2} \mathrm{O}$ treatment in the first cell cycle [34], the mechanism of which is stabilizing the microtubules, resulting in the expanded distribution of DDs. In this study, we showed that depolymerizing the microtubules by nocodazole can reverse the dorsalized phenotype caused by 0 -mpf lithium exposure, so a question arises as to whether lithium's effect on dorsal-ventral axis formation is a consequence of microtubule stabilization. Therefore we treated the wild-type embryos at 0-mpf with paclitaxel, a proved microtubule stabilizer. Interestingly, and unexpectedly, no dorsalized embryos were obtained after 0 -mpf $7.5 \mu \mathrm{g} / \mathrm{ml}$ paclitaxel exposure, and on the contrary, $10.0 \%$ $(\mathrm{n}=40)$ treated embryos showed a typical ventralized phenotype with no notochord observed at $12 \mathrm{hpf}$ (Figure $6 \mathrm{~B}, \mathrm{~A}$ as an untreated control). Other treated embryos exhibited a relatively normal dorsal-ventral axis but with mild defective convergenceextension, small head and malformed somites (Figure 6D, C as an untreated control, $\mathrm{E}$ is a ventralized embryo). We also tested the effect of paclitaxel exposure on tkk mutant embryos, and 

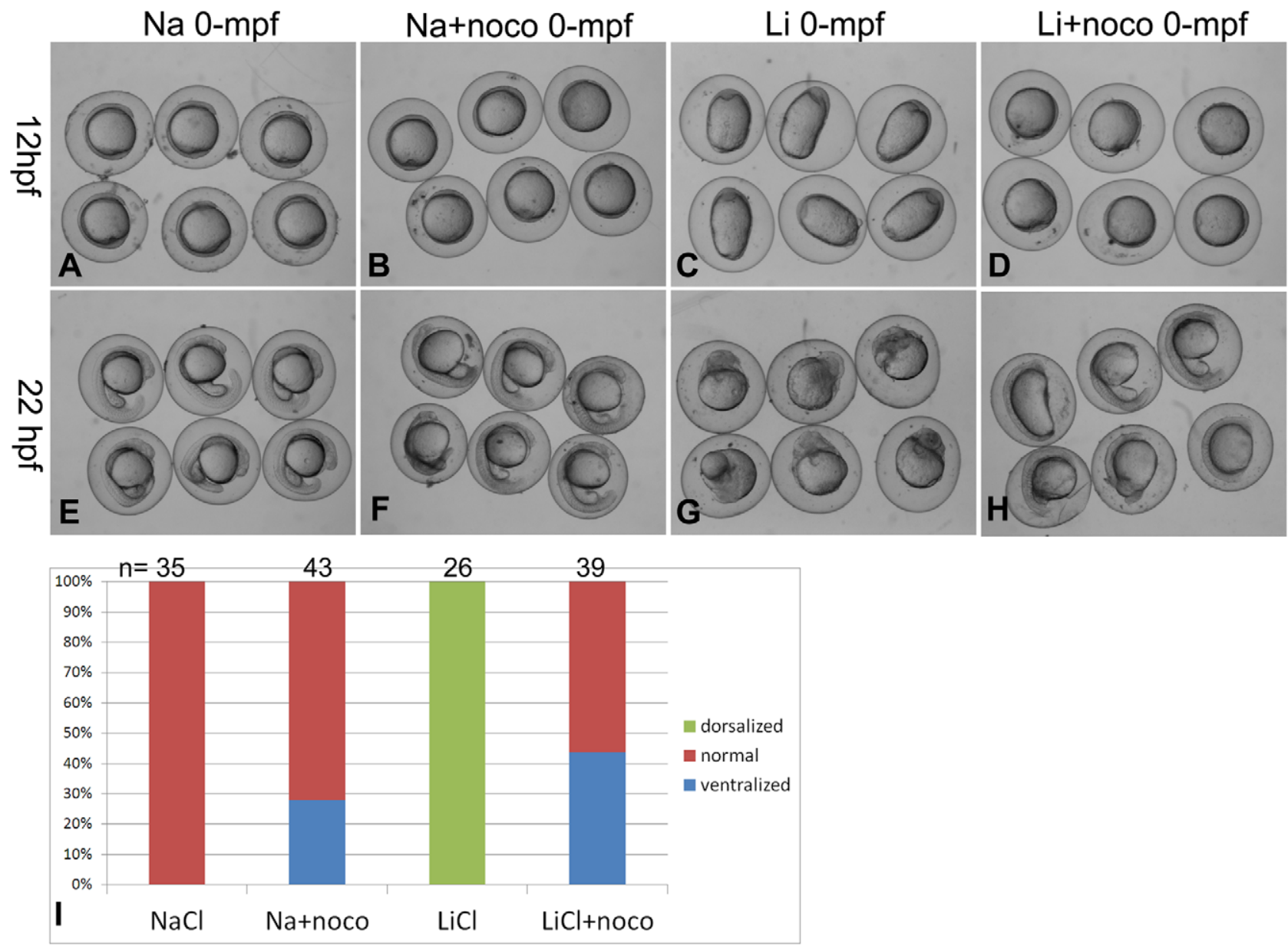

Figure 5. 0-mpf nocodazole treatment reverses the dorsalizing effect of the $\mathbf{0}$-mpf lithium treatment. The 0 -mpf embryos were treated with $0.35 \mathrm{M} \mathrm{LiCl}$ solution in the absence or presence of $0.1 \mu \mathrm{M}$ nocodazole for $5 \mathrm{~min}$, and then observed at $12 \mathrm{hpf}$ and $22 \mathrm{hpf} .035 \mathrm{M} \mathrm{NaCl}$ treatment served as control. (A and E) 0-mpf NaCl treated embryos at $12 \mathrm{hpf}(\mathrm{A})$ and $22 \mathrm{hpf}$ (E). (B and F) 0-mpf NaCl and nocodazole co-treated embryos at $12 \mathrm{hpf}(\mathrm{B})$ and $22 \mathrm{hpf}(\mathrm{F})$. (C and G) 0-mpf lithium treated embryos at $12 \mathrm{hpf}(\mathrm{C})$ and $22 \mathrm{hpf}(\mathrm{G})$. (D and H) 0-mpf lithium and nocodazole co-treated embryos at $12 \mathrm{hpf}(\mathrm{D})$ and $22 \mathrm{hpf}(\mathrm{H})$. (I) Statistical data were obtained at $12 \mathrm{hpf}$ for the experiment with embryo numbers on the top of each column. doi:10.1371/journal.pone.0036655.g005

accordingly did not observe any rescuing effect of the ventralized phenotype (Figure 6F). These results suggested that stabilized microtubules are not sufficient to mediate the dorsalizing activity of 0 -mpf lithium treatment.

\section{The dorsalizing effect of 0 -mpf lithium exposure is owing to the inhibition of GSK-3 activity}

Lithium inhibits GSK-3 and IMPase, so another important question is to distinguish which enzyme serves as the real target. To address this question, we further used much more specific chemical inhibitors including GSK-3 inhibitor IX and IMPase inhibitor L690, 330, to analyze which one can mimic the lithium treatment in the dynamic dorsalizing activity. Just like lithium treatment, nearly all the 0-mpf GSK-3 inhibitor IX treated embryos exhibited a long elliptical shape at $11.5 \mathrm{hpf}$, and the large cell aggregation at the animal pole was also observed at $26 \mathrm{hpf}$. The dorsalizing activity of 2-cell-stage GSK-3 inhibitor IX treatment became much weaker than the 0 -mpf treatment, which is also very similar to that of lithium exposure (Figure 7A, B, C, D, E, F, G, H, I, J, K, L, Y). We then injected L690, 330 or LiCl at 0 mpf zebrafish embryos and compared the resulting phenotypes. Injection of L690, 330 resulted in embryos with a typical convergence-extension (CE) defect rather than the dorsalized phenotype. These embryos appeared spherical at $11.5 \mathrm{hpf}$ and showed distinct dorsal-ventral axis at $24 \mathrm{hpf}$, although the length of the dorsal axis was much shorter. In sharp contrast, 0-mpf LiCl injected embryos only exhibited dorsalized phenotype. When the injection was performed at 2-cell stage, the dorsalizing effect lithium was reduced, however we failed to find such a phenomenon in L690, 330 injection experiment (Figure 7M, N, $\mathrm{O}, \mathrm{P}, \mathrm{Q}, \mathrm{R}, \mathrm{S}, \mathrm{T}, \mathrm{U}, \mathrm{V}, \mathrm{W}, \mathrm{X}, \mathrm{Y})$. To confirm the phenotypic data at the molecular level, we performed in situ hybridization experiment on $50 \%$ epiboly embryos using the probe of gsc. As expected, most of the 0-mpf GSK-3 inhibitor IX and 0-mpf lithium treated embryos showed radial or expanded expression of gsc. However, all of the L690, 330 treated embryos showed a relatively normal expression pattern (Figure $7 \mathrm{Za}-\mathrm{Zf}$ ). These data demonstrated that the 0-mpf treatment of GSK-3 inhibitor IX but not L690, 330 can mimic lithium in the dorsalizing activity, which strongly suggested that GSK-3 activity is required for the dorsalventral specification shortly after fertilization. 

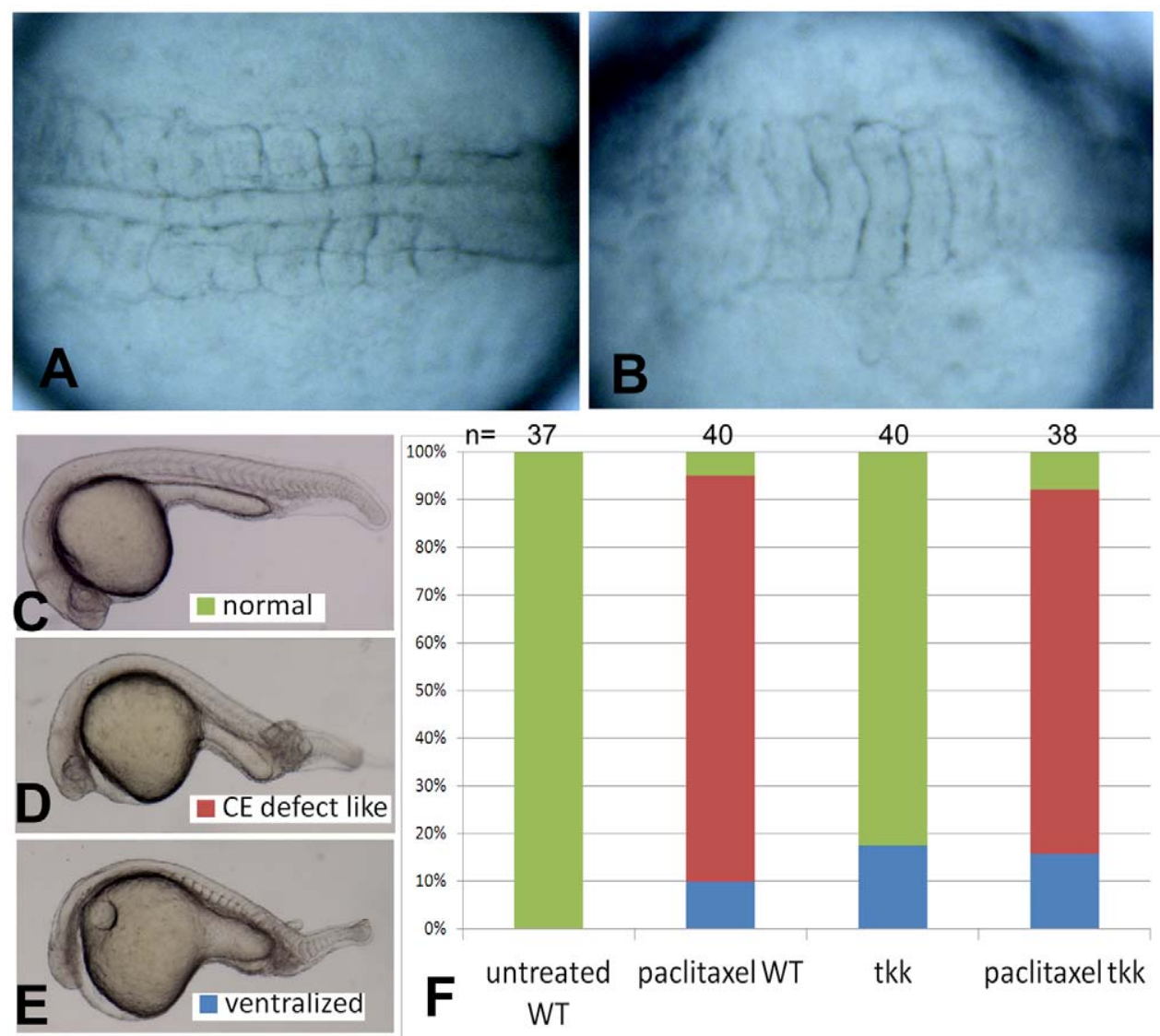

WT

Figure 6. Paclitaxel treatment on wild-type and $\boldsymbol{t} \boldsymbol{k} \boldsymbol{k}$ mutant embryos. (A) Dorsal view of an untreated $12 \mathrm{hpf}$ embryo. (B) Dorsal view of a 0mpf paclitaxel treated embryo with ventralized phenotype. (C) An embryo with normal phenotype. (D) An embryo with CE defect like phenotype, showing a smaller head, shorter anterior-posterior axis and malformed somites. (E) A ventralized embryo. Embryos in (C-E) were observed at $24 \mathrm{hpf}$. The statistical data based on the $24 \mathrm{hpf}$ observation were shown in (F) with embryo numbers shown on the top of each column. doi:10.1371/journal.pone.0036655.g006

\section{0-mpf GSK-3 inhibition disrupts the parallel pattern of cortical microtubules and the polarized migration of Wnt8a transcripts}

It was reported that destroying the assembly of the microtubules by chemical or physical factors can ventralize vertebrate embryos $[12,14]$. Blocking the microtubule dependent transport of DDs can also generate ventralized phenotype [21]. However, 0-mpf lithium treatment acts in an opposite way to generate the dorsalized phenotype, so it is impossible that 0 -mpf lithium treatment can disrupt the assembly of cortical microtubules or inhibit the DDs transport. In fact, 0-mpf lithium treatment may affect the transport and broaden the distribution of DDs by influencing the remodeling of cortical microtubules after fertilization. To test this possibility, we stained and visualized the vegetal microtubule arrays formed $\sim 20 \mathrm{mpf}$ by confocal microscopy. As reported, well-formed parallelly distributed microtubules in the vegetal pole region were detected in $\mathrm{NaCl}$ treated embryos (Figure 8A,), but in sharp contrast, the orientation of microtubules in 0 -mpf lithium and GSK-3 inhibitor IX treated embryos were randomized and organized like an irregularly weaved net, and the microtubule bundles appeared much thinner with respect to control (Figure 8B, C). We further tested if the polarized migration of a recently identified DD, Wntoa mRNA, was affected by GSK-3 inhibition. In 0 -mpf $\mathrm{NaCl}$ treated 4-cell stage embryos, almost all the embryos $(92.6 \%, \mathrm{n}=27)$ tested showed a biased distribution of Wnt8a in the yolk cortex (Figure 8D), but after inhibiting GSK-3 at 0-mpf by lithium or GSK-3 inhibitor IX, this asymmetric pattern disappeared, instead, the distribution of Wnt8a transcripts became much more smearing (Figure $8 \mathrm{E}$ and $\mathrm{F}$ ). We also examined the expression of Wnt8a in 4-cell stage tkk mutant embryos, and found that in most cases $(93.8 \%, \mathrm{n}=48)$, Wnt8a transcripts were restricted to the vegetal pole with indistinguishable biased distribution, and 0-mpf lithium treatment or GSK-3 inhibitor IX treatment did not alter their distribution in this mutant (Figure 8G, $\mathrm{H}, \mathrm{I})$, which was consistent with the previous data that 0 -mpf lithium treatment failed to reverse the ventralized phenotype of $t k k$ mutant. These observations suggest that GSK-3 activity is critical for the parallel alignment of the microtubule arrays after fertilization, and the dorsalizing activity of 0-mpf GSK-3 inhibition is probably the consequence of randomized microtubule arrays, which lead the DDs to a much broader area of the perspective marginal zone.

\section{Discussion}

\section{Lithium can dorsalize zebrafish embryos in two completely different ways}

In this work, we have characterized another lithium-sensitive window to cause dorsalization of the zebrafish embryos. Lithium treatment carried out at the late cleavage stage was previously described for its capability to induce dorsalization in Xenopus as well as in zebrafish [29,35-38]. However, the mechanisms of the 
$\mathrm{NaCl}$

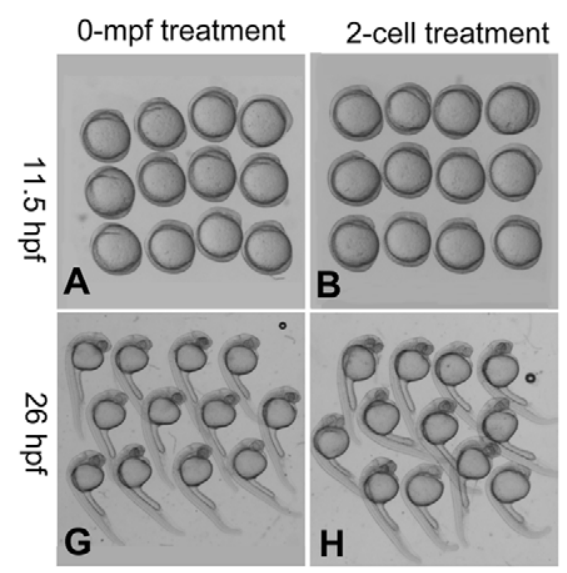

$\mathrm{NaCl}$

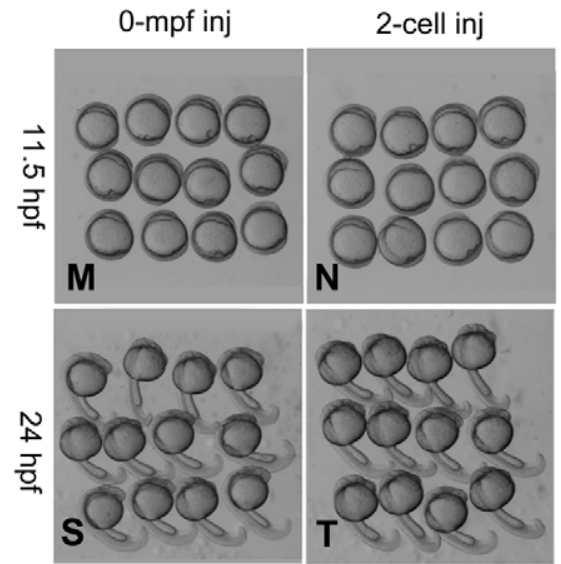

$\mathrm{LiCl}$

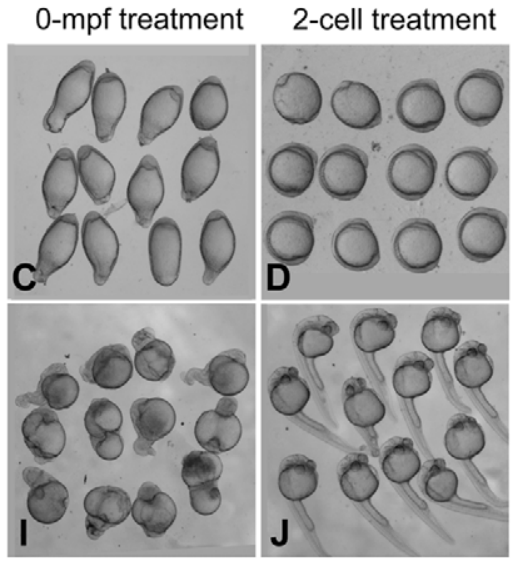

$\mathrm{LiCl}$

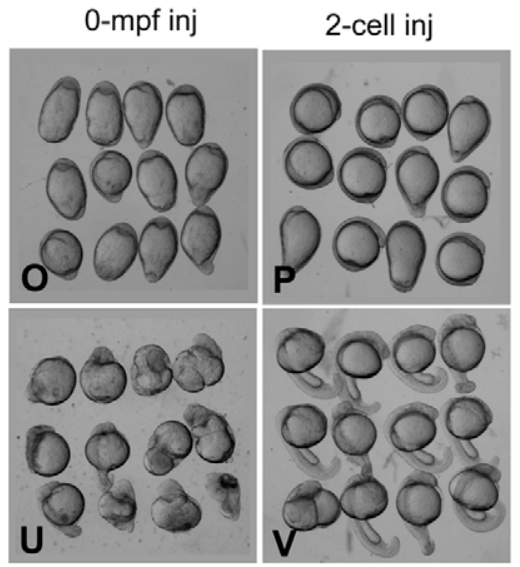

GSK-3 inhibitor IX

0-mpf treatment 2-cell treatment

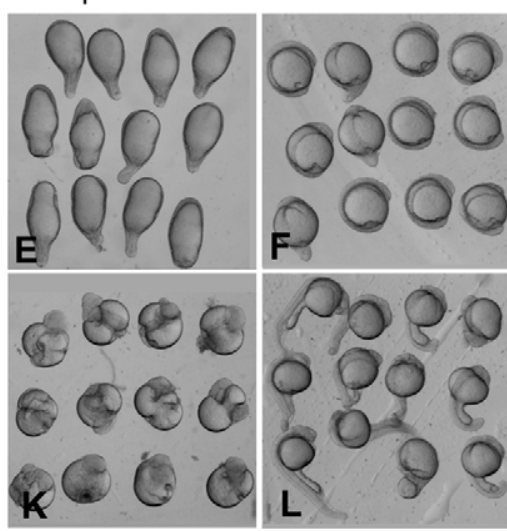

L690,330

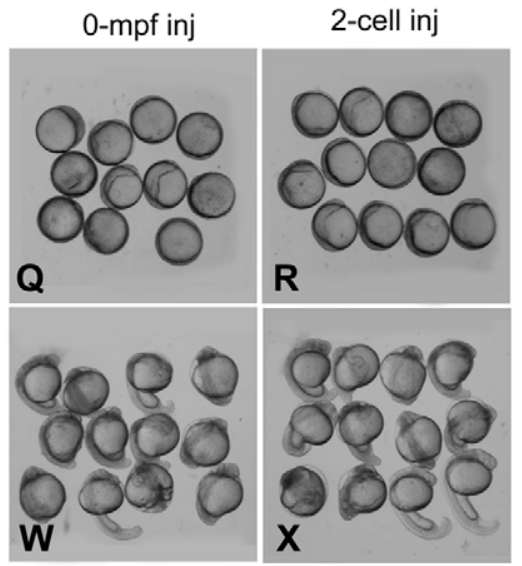

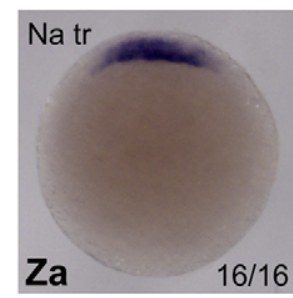

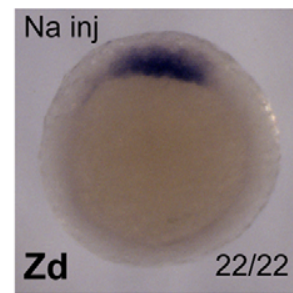

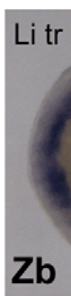

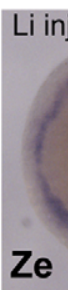

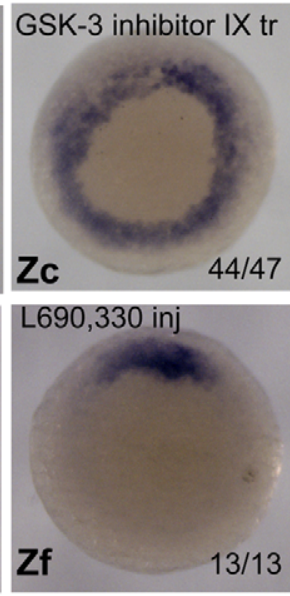

Figure 7. Comparison between lithium and chemical inhibitors of GSK-3 or IMPase in the dorsalizing activity. (A-L) Phenotypic comparison of $\mathrm{NaCl}$, lithium and GSK-3 inhibitor IX exposures. (M-X) Phenotypic comparison of NaCl, lithium and L690, 330 injections. Embryos in A-X were observed at stages indicated at the left side of the figure. $(Y)$ Statistical data of the phenotypic analysis at 11.5 hpf with embryo numbers on the top of each column. (Za-Zf) Examination of gsc expression in 0-mpf NaCl, LiCl, GSK-3 inhibitor IX and L690,330 exposed (Za-Zc) or injected embryos (Zd-Zf).

doi:10.1371/journal.pone.0036655.g007

dorsalizing effect of lithium treatment in the early (SW1) and late (SW2) sensitive windows are completely different. Lithium treatment at SW2 was reported to inhibit GSK-3, a negative regulator of Wnt/ $\beta$-catenin pathway, thus can directly dephosphorylate and stabilize $\beta$-catenin, and activate the expression of the downstream dorsal organizer genes [28,39]. 32-cell-stage 


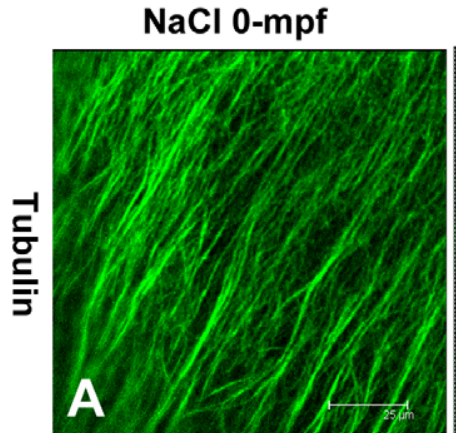

$11 / 11$

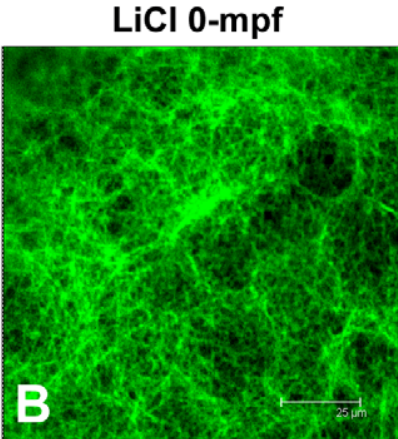

$8 / 8$

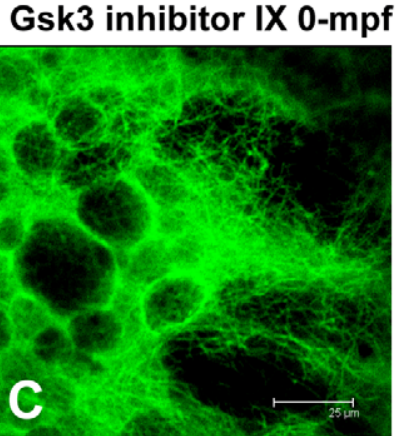

$10 / 11$

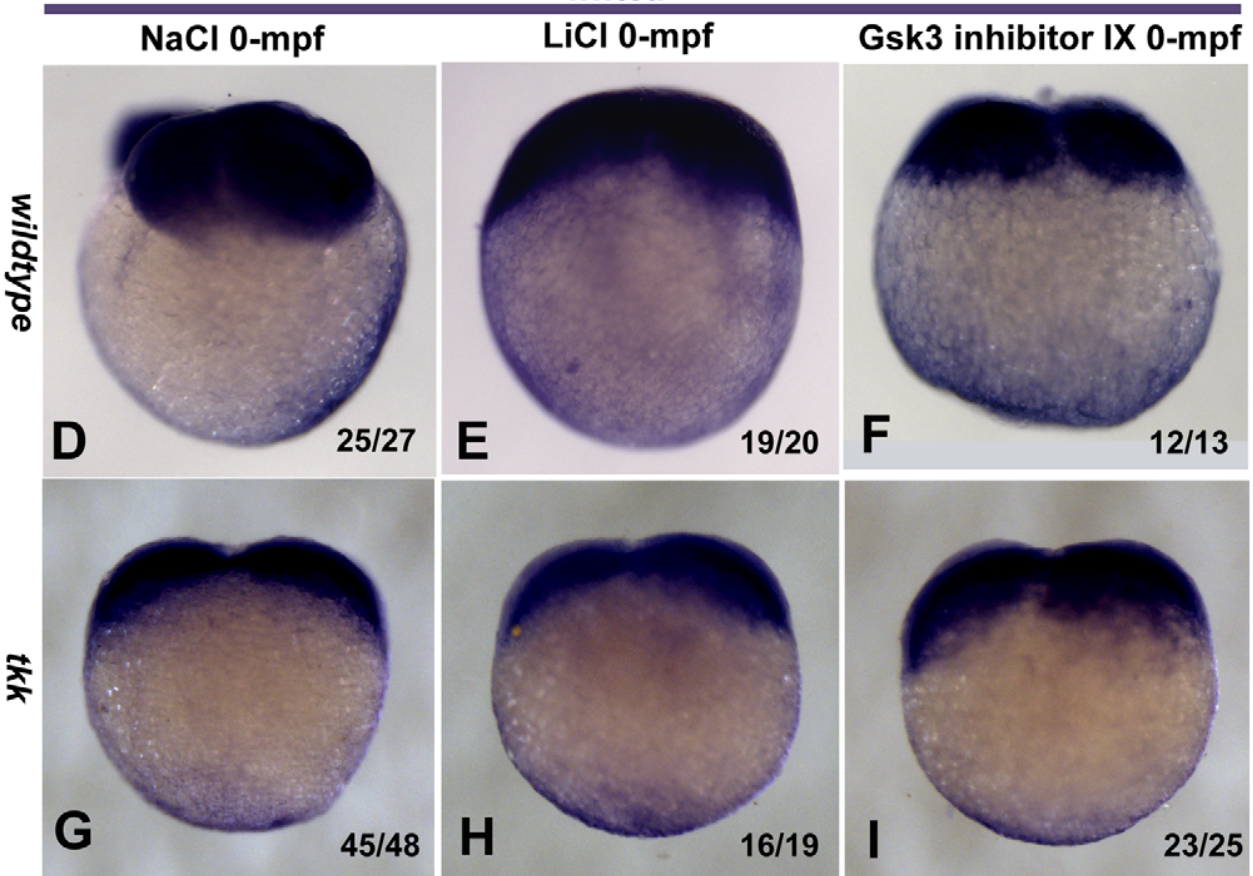

Figure 8. 0-mpf inhibition of GSK-3 activity randomized the parallel microtubule arrays at the vegetal pole and the biased migration of Wnt8a transcripts. Microtubule staining with an anti- $\beta$-tubulin antibody to visualize the microtubule arrays formed at around $20 \mathrm{mpf}$ at the vegetal pole. (A) Parallel microtubule arrays detected in $\mathrm{NaCl}$ treated embryos. (B) Randomized aligned microtubule arrays detected in 0-mpf lithium treated embryos. (C) Similar phenomenon detected in 0-mpf GSK-3 inhibitor treated embryos. (D-F) 0-mpf lithium and GSK-3 inhibitor IX treatments disrupted the polarized distribution of Wnt8a mRNA observed at 4-cell stage. (D) A 0-mpf NaCl treated wild-type embryo, (E) A 0-mpf lithium treated wild-type embryo, (F) A 0-mpf GSK-3 inhibitor IX treated wild-type embryo (G) Wnt8a mRNA restricted to the animal pole region of the $0-\mathrm{mpf} \mathrm{NaCl}$ treated 4-cell-stage $t k k$ mutant embryos. (H I) Exposure of GSK-3 inhibitors failed to alter the distribution of Wnt8a mRNA in 4-cell-stage tkk mutant embryos.

doi:10.1371/journal.pone.0036655.g008

lithium treatment can efficiently rescue or even reverse the ventralized phenotype caused by DDs deficiency in Xenopus and in zebrafish, indicating that the mechanism is downstream of the dorsal determinants (DDs) [36] (this study). If the dorsalizing activity of the 0-mpf lithium treatment functions through the same mechanism, these ventralized embryos should also be rescued. In fact, our data led us in an opposite conclusion that 0-mpf lithium treatment was unable to rescue the ventralized embryos by $t k k$ mutation or nocodazole treatment (Fig 3, 4, 5). These results demonstrated that lithium treatment performed in SW1 and SW2 should impose their dorsalizing effect via different ways. This difference can further be sensed in two other phenomena: first, it seemed that zebrafish embryos in SW1 are more sensitive to lithium than in SW2. Slightly increasing treatment time $(10 \mathrm{~min}$ e.g.) at 0 -mpf often blocked or disrupted cell division and led to lethality before gastrula stage (data not shown), which is not the case for the 32-cell-stage lithium treatment. Second, the induction of dorsal organizer gene $g_{s c}$ by lithium treatment at SW1 or SW2 is different. This gene is normally expressed in an integrated manner in the dorsal marginal zones; 0-mpf lithium treatment often enlarged the expression region but made the positive cells discrete, while in contrast, 32-cell-stage lithium treatment caused a smoothly enlarged gsc expression region (Fig 4). This difference may be explained by the possibility that lithium directly activates Wnt/ $\beta$-catenin pathway in the whole embryo in SW2, inducing gsc expression in all the marginal cells, but in SW1, lithium acts in a totally different way and the mosaic $g s c$ expression is indirectly caused by a certain upstream mechanism.

The SW1 here is similar to the sensitive window of brief nocodazole treatment, in which the ventralized zebrafish embryos 


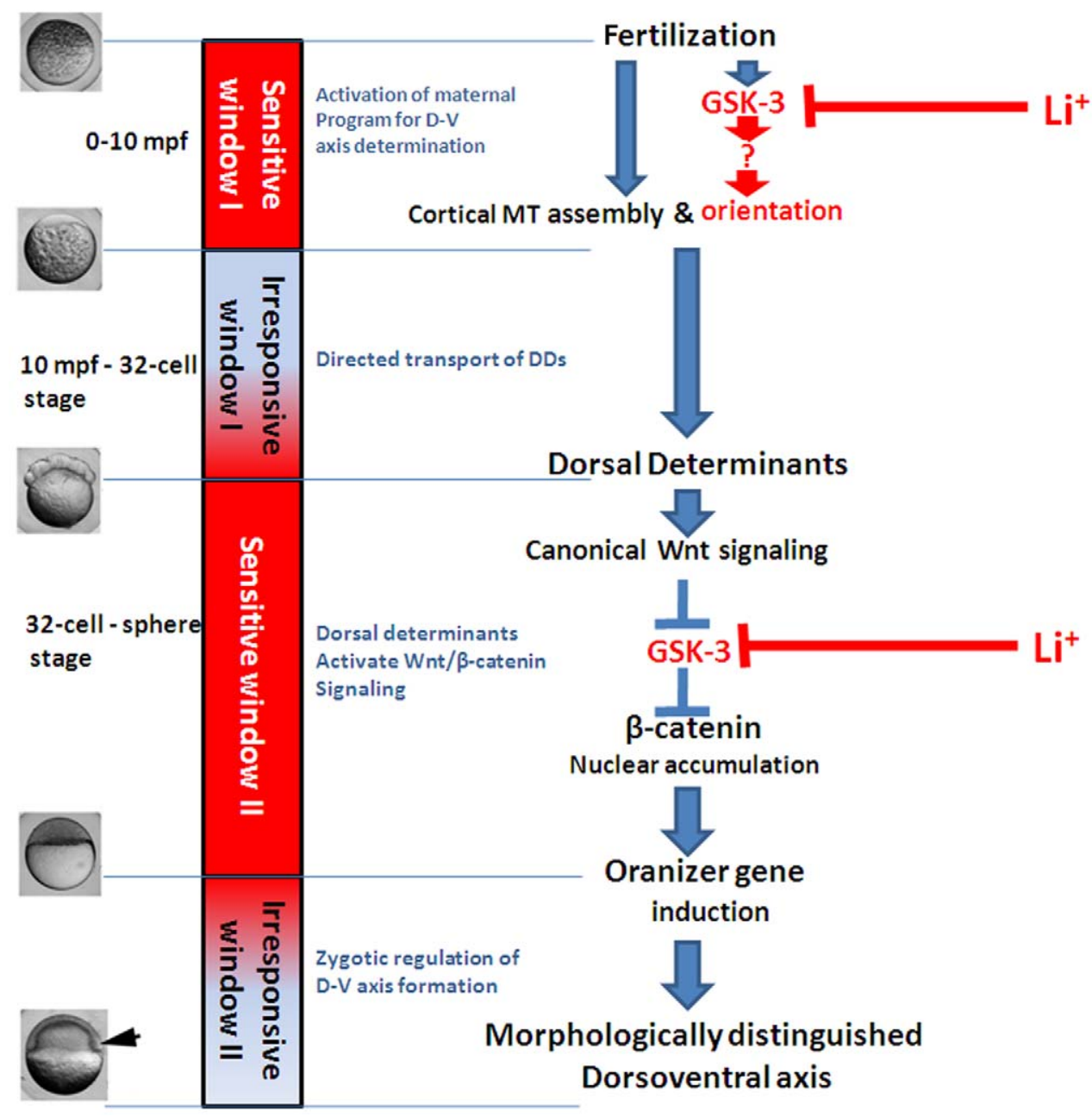

Figure 9. Model of dorsal-ventral axis formation in zebrafish. The zebrafish DV axis specification can be divided to four phases based on the dynamic alteration of the dorsalizing activity of lithium treatment. SW1 designates $0-10 \mathrm{mpf}$ in which lithium treatment can cause dorsalization of the embryos. In this phase, fertilization initiates a GSK-3 dependent mechanism regulating the orientation but not stablization of vegetal microtubules which is critical for the dorsalward transport of DDs like Wnt8a mRNA. UW1 designates the first unresponsive window of lithium treatment, from $10 \mathrm{mpf}$ to the 32-cell stage, in which, especially in the early period, lithium treatment fails to efficiently cause dorsalization. In this period, dorsal determinants are transported from the vegetal pole to the perspective dorsal side. The transduction of Wnt/ $\beta$-catenin pathway is probably blocked by some unknown mechanism in SW1 and UW1. SW2 designates the period from the 32-cell stage to the mid-blastula stage. In this period, dorsally located DDs are able to inhibit GSK-3, causing the stabilization and nuclear localization of $\beta$-catenin, and lead to the expression of dorsal organizer genes. In UW2, lithium treatment loses its ability to dorsalize zebrafish embryos and the organizer gene expression is translated gradually by cell movement to morphologically distinguished dorsal-ventral axis. Arrow head at the lower-left corner indicates the shield. doi:10.1371/journal.pone.0036655.g009

can be generated when nocodazole treatment is performed before $10 \mathrm{mpf}$, but the embryos were seldom ventralized when treated at $15 \mathrm{mpf}[12]$. As nocodazole treatment inhibits the microtubule polymerization, the similarity between SW1 and ventralizing window of nocodazole treatment may suggest that lithium also imposes its effect on the microtubule organization, but in a very different way. In this study, we presented three lines of evidence in support of this hypothesis. First, in the presence of nocodazole, the dorsalizing activity of the 0 -mpf lithium treatment was completely lost (Figure 5), and the embryos only showed the ventralized phenotype, i.e. nocodazole treatment can override the lithium treatment, suggesting that the dorsalizing activity of 0 -mpf lithium treatment requires the normal polymerization of microtubules. Second, 0-mpf lithium treatment was unable to dorsalize the maternal mutant tokkaebi (tkk) zebrafish embryos which harbors a mutation in the syntabulin gene encoding a protein required for the cargo transport along microtubule arrays [13]. Third, and most directly, we showed that the parallel microtubule array formed $\sim 20 \mathrm{mpf}$ in the vegetal pole region was randomized by the $0-\mathrm{mpf}$ lithium treatment, which raised a possibility that after 0 -mpf lithium treatment, the transport of DDs is not directed to the perspective dorsal region but to the circumference of the margin, and this was further confirmed by marking a newly identified dorsal determinant, Wnt8a mRNA. These results thus put the mechanism of the 0 -mpf lithium treatment at the upstream level to the microtubule dependent transport of DDs.

2. Wnt/ $\beta$-catenin independent GSK-3 activity is required for the dorsal-ventral axis formation

GSK-3 and inositol monophosphatase (IMPase) are proved targets of lithium [25]. Our study using specific chemical inhibitors demonstrates GSK-3 as the real target of 0 -mpf lithium treatment. GSK-3 plays an important role in Wnt/ $\beta$-catenin signaling. 
However, our data indicate that directly activating $\mathrm{Wnt} / \beta$-catenin pathway cannot be the cause of the dorsalizing activity of lithium treatment in SW1. In fact, GSK-3 inhibitors can randomize the alignment of vegetal cortical microtubule arrays and disturb the biased transport of the Wnt $8 a$ transcripts initiated by fertilization. Based on these observations, the dorsalizing effect of 0-mpf GSK-3 inhibition can be properly interpreted. In normal embryos, the active GSK-3 shortly after fertilization may facilitate the formation of parallelly aligned microtubule arrays, which is essential for the polarized transport of Wntoa mRNA. But the polymerized microtubules failed to form parallel bundles after GSK-3 inhibition, instead, randomized and much thinner microtubule filament formed after lithium or GSK-3 inhibitor IX treatment. These net-like microtubule arrays can still transport DDs like Wnto a transcripts, but might lead them to migrate in all the directions across the yolk cortex, so that many ventral lateral marginal cells receive sufficient dorsal-determining signals to change their fate. This deduction was supported by the fact that gsc positive cells often discretely distributed around the margin in 0mpf lithium or GSK-3 inhibitor IX treated embryos. Syntabulin was thought as a linker between DDs and Kinesin motors during the microtubule dependent transport, so lacking this protein can reduce the amount of DDs transported to the dorsal margin (Wnt8a transcripts still located at the vegetal pole in 4-cell stage $t k k$ embryos shown in Figure 8G), and causes ventralized phenotype. It can be imagined that the reduced DDs successfully transported in tkk embryos will be further diluted after their randomized migration caused by GSK-3 inhibition, and this dilution will make a sub-threshold supply of DDs for more marginal cells resulting in a more frequent appearance of ventralized embryos.

Data in this study also raised a question of whether GSK-3 regulates any microtubule-related protein independent of the Wnt/ $\beta$-catenin signaling shortly after fertilization. It has been established that the microtubule associated protein Tau and MAP1B are substrate of GSK-3 $\beta$ (reviewed by [24]). Although no literature deals with the role that these proteins plays in the early $\mathrm{D}-\mathrm{V}$ axis formation, it has been well studied that Tau is essential for microtubule stabilization in neuronal axons. Hyperphosphorylation and intracellular fibrillar formation of tau protein deter its ability to bind to and stabilize microtubules, and is a pathology found in Alzheimer's disease [40,41]. Lithium is able to reduce the amount of phosphorylated Tau in cell culture [39], so it is conceivable that lithium may activate too much Tau by inhibiting the activity of GSK-3 after fertilization and randomize the vegetal paralleled microtubule arrays. This hypothesis, however, is far from being solidified. First, whether Tau owns a maternal expression is not verified. Second, our data showed that only stabilizing microtubules by paclitaxel treatment was not sufficient to dorsalize zebrafish embryos like GSK-3 inhibitors. Beside these doubts, another interesting aspect is that GSK-3 seemed to function oppositely in tkk mutant embryos for the unexpected ventralizing effect of lithium treatment. This phenomenon might indicate that the GSK-3 regulated process is extremely sensitive to slight disturbance of the cargo transport system, and the Syntabulin protein might functionally interact with GSK-3 or its substrate. Thus, further investigation is required to identify GSK-3 substrates responsible for the short-lived microtubule remodeling process. Functional analysis of these unknown molecules might shed light on the mystery of the instantaneous SW1 and the anomalous behavior of lithium treatment on $t k k$ embryos.

\section{The unresponsive window (UW) of lithium treatment during the early cleavage stage}

It is very interesting that lithium had almost no effect on zebrafish embryos when treatment was performed during the first 1-2 cell cycles [29] (this study). Wnt signaling is not activated until midblastula stage, as revealed by TopdGFP transgenic zebrafish [42]. Our data also suggest that Wnt/ $\beta$-catenin signaling cannot be efficiently activated by lithium treatment from fertilization to the late cleavage stage. There might be two possibilities for the existence of the unresponsive window: one is that the extra $\beta$ catenin stabilized by lithium treatment is degraded by an unknown negative feedback loop in the time span between the treatment and mid-blastula stage when Wnt signaling begins to activate, and the second possibility is that lithium treatment in this UW cannot rescue $\beta$-catenin from GSK-3. For the second possibility, we hypothesize that before the 32-cell stage, GSK-3 and its substrate $\beta$-catenin might be separated, and importantly at the same time, the average concentration of $\beta$-catenin is controlled below the activation threshold of the downstream cascade of Wnt signaling. GSK-3 is shown to be sequestered into the multi vesicular endosomes (MVB) in response to Wnt activation, and this phenomenon is required for the secondary axis induction in Xenopus embryos by Wnt ligands overexpression [43]. According to this, it is conceivable that GSK-3 and its substrate $\beta$-catenin might be segregated from each other by structures like MVB in the SW1 and UW, and GSK-3 is possible to be released gradually to the cytosol when cleavage continues under an unknown mechanism. According to this assumption, the existence of the unresponsive window can be properly interpreted: before the 32-cell stage, as very limited $\beta$-catenin can meet GSK-3, the lithium treatment in this stage can only increase a very small amount of $\beta$-catenin, which is not sufficient to activate organizer genes around the margin at mid-blastula stage. However, after 32-cell stage, as most $\beta$-catenin can contact the freed GSK-3 in the lateral-ventral regions, lithium treatment can stabilize large amount of $\beta$-catenin which may break the threshold to activate downstream target genes. To test these two models, more work is needed to verify the fluctuation of $\beta$-catenin before the 32-cell stage in control and lithium treated embryos, and to test if the maternal mutant with MVB formation defect is associated with the $\mathrm{D}-\mathrm{V}$ axis formation problem in the zebrafish embryos, and if GSK-3 localizes in MVB before the late cleavage stage and is released to cytosol after the 32-cell stage.

\section{Distinct phases of the dorsal-ventral axis specification revealed by lithium treatment, and remaining questions}

Based on the data in this study, we could divide the $\mathrm{D} \mathrm{V}$ axis formation process into four phases: 1) 0 -mpf to 10 -mpf, identical to the SW1, in this phase, fertilization induces a GSK-3 dependent mechanism that determines the orientation of vegetal microtubule arrays; 2) 10-mpf to 32-cell stage, corresponding to the UW1, in this phase, the dorsal determinants move directionally to the perspective dorsal marginal zone along paralleled microtubule arrays. 3) 32-cell stage to mid-blastula stage (SW2). Dorsal determinants begin to inhibit GSK-3 in the dorsal marginal zone, leading to $\beta$-catenin stabilization and nuclear localization, which further activate the early marker of the organizer; 4) Mid-blastula to early gastrula stage (UW2), in this phase, lithium treatment tends to induce posteriorization of neural system but not dorsalization. The dorsal-ventral axis specification is accomplished at the molecular level, and the asymmetric expression of dorsalventral genes is gradually translated to the morphologically distinct dorsal organizer structure-the shield (Figure 9). 
So far, we know very little about what happens in the first two stages. Specifically, we know little about the signaling cascade that initiates and directs the transport of dorsal determinants; and we have not perceived the reason why GSK-3 inhibition cannot efficiently activate $\mathrm{Wnt} / \beta$-catenin signaling in SWl and UW1. More genetic and functional work is needed to identify and analyze maternal mutants with dorsal-ventral axis defect, which may shed light on these questions, and help to understand this earliest, vital, and intricate patterning process.

\section{Materials and Methods}

\section{Ethic statement}

All embryos were handled according to relevant national and international guidelines. The study was approved by the Committee on the Ethics of Animal Experiments of Shandong University (Permit number: ECAESDUSM 2009035).

\section{Fish Strains}

Wild-type and the mutant tokkeabi (tkk) fish were used. The maternal mutant $t k k$ embryos were generated by crossing wild-type male with $t k k$ homogeneous female. The penetrance was evaluated by observing the phenotype at $24 \mathrm{hpf}$. The tokkeabi mutant strain is a gift from Dr. Hibi.

\section{Egg collection, lithium, nocodazole, paclitaxel and GSK-3 inhibitor IX exposure}

To ensure synchronic development of the zebrafish embryos, eggs were collected immediately when the female spawned. The fish mated in a chamber made up of three parts, an outer tank, an inner tank with narrow slits that allow the eggs to fall through to the outer tank, and a plastic sheet set in the middle of the inner tank to separate the male and female fish before mating. We kept a pair of fish separated in this chamber overnight and prepared an extra outer tank with egg water before the experiment. On the morning of the next day, the plastic sheet was removed and the fish began to mate. Once the female began to spawn eggs, the male and female were quickly separated by the plastic sheet and the inner tank was immediately transferred to the prepared extra outer tank. The synchronic eggs were collected immediately from the original outer tank and subjected to treatment or injection at indicated stages. Another batch of synchronic eggs can be collected by removing the plastic sheet again when convenient and repeating the procedure described above. In order to get sufficient synchronic eggs, the male and female fish were raised separately for more than a week before the experiment, and the female normally releases 20-40 eggs at one time during the mating. The data was obtained by several treatments with different batches of synchronic eggs. The collected embryos were treated with $0.3 \mathrm{M} \mathrm{LiCl}$ solution (diluted in E3 buffer) for $8 \mathrm{~min}$ at indicated stages. The nocodazole (Sigma, M1404-2MG) were dissolved in DMSO at a concentration of $5 \mathrm{mg} / \mathrm{ml}$, and dilute in E3 buffer or mixed with the $0.35 \mathrm{M} \mathrm{LiCl}$ solution at a final concentration of $0.1 \mu \mathrm{g} / \mathrm{ml}$. The 0 -mpf treatment of $\mathrm{LiCl}$,

\section{References}

1. Langdon YG, Mullins MC (2010) Maternal and Zygotic Control of Zebrafish Dorsoventral Axial Patterning. Annu Rev Genet.

2. De Robertis EM (2006) Spemann's organizer and self-regulation in amphibian embryos. Nat Rev Mol Cell Biol 7: 296-302.

3. Hibi M, Hirano T, Dawid IB (2002) Organizer formation and function. Results Probl Cell Differ 40: 48-71.

4. Harland R, Gerhart J (1997) Formation and function of Spemann's organizer. Annu Rev Cell Dev Biol 13: 611-667. nocodazole or $\mathrm{LiCl} /$ nocodazole mixture was carried out immediately when the female fish spawned. As the embryos were extremely sensitive to nocodazole, so the treated time was reduced to $5 \mathrm{~min}$, which is sufficient for $0.35 \mathrm{M} \mathrm{LiCl}$ to induce dorsalization. Paclitaxel (Sigma, T7191-5MG) was dissolved in DMSO at $1 \mathrm{mg} / \mathrm{ml}$, then diluted to $7.5 \mu \mathrm{g} / \mathrm{ml}$ in an E3 buffer based solution containing $0.5 \mathrm{mg} / \mathrm{ml}$ Pronase (Roche, 11459643 001), and the embryos were treated in this solution for $8 \mathrm{~min}$. GSK-3 inhibitor IX (Santa Cruz, sc-202634) was dissolved in DMSO at $10 \mathrm{mg} / \mathrm{ml}$ as a stock solution, and the embryos were exposed in a working solution of $10 \mu \mathrm{g} / \mathrm{ml}$ containing $0.5 \mathrm{mg} / \mathrm{ml}$ Pronase in E3 buffer for $8 \mathrm{~min}$. The treated embryos were rinsed in E3 buffer for three times and incubated at $28.5^{\circ} \mathrm{C}$ until observation and fixation.

\section{Microinjection}

$\mathrm{LiCl}$ and $\mathrm{NaCl}$ were diluted in deionized water at a concentration of $0.15 \mathrm{M}$ respectively, and was injected $2 \mathrm{nl}$ to the 0-5 mpf and 2-cell-stage embryos. 2.5 mM L690, 330 (Santa Cruz, sc-202685A) aqueous solution was injected $2 \mathrm{nl}$ for each embryo at the same stage to those used in $\mathrm{LiCl}$ and $\mathrm{NaCl}$ injections (the concentration of L690, 330 was used according to previous studies $[44,45])$. The microinjection was performed using the MPPI-3 Pressure Injector.

\section{Whole mount in situ hybridization}

Sequences of goosecoid (gsc), eve1 and Wnt8a were cloned in pGEM-Teasy vector. Anti-sense RNA probes were synthesized using the digoxigenin-UTP (DIG) in vitro transcription kit (Roche Applied Science, Indianapolis, IN, USA). Whole-mount in situ hybridization was conducted according to the zebrafish book [46].

\section{Antibodies, immunofluorescence and confocal microscopy}

The $\beta$-catenin localization was visualized by using an antibody from Abcam (ab6302), and the whole mount immunofluorecence was performed in a routine way as described [47], and the focal plane was selected near the margin; The microtubule was stained by an anti- $\beta$-tubulin antibody (Chemicon KMX-1), and the protocol were previously described $[13,48]$. The focal planes were selected near the vegetal pole. The images were taken under a $10 \mathrm{x}$ objective of a Leica TCS SP2 confocal microscope.

\section{Acknowledgments}

We are grateful to Dr. Hibi Masahiko, Bioscience and Biotechnology Center, Nagoya University, Japan, for presenting tokkeabi mutant strain, and helpful discussions. We also thank Dr. Shi De-li for critical reading of the manuscript.

\section{Author Contributions}

Conceived and designed the experiments: MS HZ. Performed the experiments: MS YL ZL YZ LW. Analyzed the data: MS YL CL HZ. Wrote the paper: MS HZ.

5. De Robertis EM (2009) Spemann's organizer and the self-regulation of embryonic fields. Mech Dev 126: 925-941.

6. Schier AF, Talbot WS (2005) Molecular genetics of axis formation in zebrafish. Annu Rev Genet 39: 561-613.

7. Driever W (1995) Axis formation in zebrafish. Curr Opin Genet Dev 5: 610-618.

8. Hainski AM, Moody SA (1992) Xenopus maternal RNAs from a dorsal animal blastomere induce a secondary axis in host embryos. Development 116: 347-355. 
9. Weaver C, Farr GH 3rd, Pan W, Rowning BA, Wang J, et al. (2003) GBP binds kinesin light chain and translocates during cortical rotation in Xenopus eggs. Development 130: 5425-5436.

10. Miller JR, Rowning BA, Larabell CA, Yang-Snyder JA, Bates RL, et al. (1999) Establishment of the dorsal-ventral axis in Xenopus embryos coincides with the dorsal enrichment of dishevelled that is dependent on cortical rotation. J Cell Biol 146: 427-437.

11. Kimmel CB, Ballard WW, Kimmel SR, Ullmann B, Schilling TF (1995) Stages of embryonic development of the zebrafish. Dev Dyn 203: 253-310.

12. Jesuthasan S, Stahle U (1997) Dynamic microtubules and specification of the zebrafish embryonic axis. Curr Biol 7: 31-42.

13. Nojima H, Rothhamel S, Shimizu T, Kim CH, Yonemura S, et al. (2010) Syntabulin, a motor protein linker, controls dorsal determination. Development 137: 923-933.

14. Strahle U, Jesuthasan S (1993) Ultraviolet irradiation impairs epiboly in zebrafish embryos: evidence for a microtubule-dependent mechanism of epiboly. Development 119: 909-919.

15. Ober EA, Schulte-Merker S (1999) Signals from the yolk cell induce mesoderm, neuroectoderm, the trunk organizer, and the notochord in zebrafish. Dev Biol 215: $167-181$.

16. Mizuno T, Yamaha E, Kuroiwa A, Takeda H (1999) Removal of vegetal yolk causes dorsal deficencies and impairs dorsal-inducing ability of the yolk cell in zebrafish. Mech Dev 81: 51-63.

17. Lu FI, Thisse C, Thisse B (2011) Identification and mechanism of regulation of the zebrafish dorsal determinant. Proc Natl Acad Sci U S A 108: 15876-15880.

18. Dougan ST, Warga RM, Kane DA, Schier AF, Talbot WS (2003) The role of the zebrafish nodal-related genes squint and cyclops in patterning of mesendoderm. Development 130: 1837-1851.

19. Schneider S, Steinbeisser H, Warga RM, Hausen P (1996) Beta-catenin translocation into nuclei demarcates the dorsalizing centers in frog and fish embryos. Mech Dev 57: 191-198.

20. Bellipanni G, Varga M, Maegawa S, Imai Y, Kelly C, et al. (2006) Essential and opposing roles of zebrafish beta-catenins in the formation of dorsal axial structures and neurectoderm. Development 133: 1299-1309.

21. Kelly C, Chin AJ, Leatherman JL, Kozlowski DJ, Weinberg ES (2000) Maternally controlled (beta)-catenin-mediated signaling is required for organizer formation in the zebrafish. Development 127: 3899-3911.

22. Xiong B, Rui Y, Zhang M, Shi K, Jia S, et al. (2006) Tobl controls dorsal development of zebrafish embryos by antagonizing maternal beta-catenin transcriptional activity. Dev Cell 11: 225-238.

23. Nojima H, Shimizu T, Kim CH, Yabe T, Bae YK, et al. (2004) Genetic evidence for involvement of maternally derived Wnt canonical signaling in dorsal determination in zebrafish. Mech Dev 121: 371-386.

24. Gould TD, Manji HK (2005) Glycogen synthase kinase-3: a putative molecular target for lithium mimetic drugs. Neuropsychopharmacology 30: 1223-1237.

25. Shaldubina A, Agam G, Belmaker RH (2001) The mechanism of lithium action: state of the art, ten years later. Prog Neuropsychopharmacol Biol Psychiatry 25: 855-866.

26. Gurvich N, Klein PS (2002) Lithium and valproic acid: parallels and contrasts in diverse signaling contexts. Pharmacol Ther 96: 45-66.

27. Ryves WJ, Harwood AJ (2001) Lithium inhibits glycogen synthase kinase-3 by competition for magnesium. Biochem Biophys Res Commun 280: 720-725.

28. Klein PS, Melton DA (1996) A molecular mechanism for the effect of lithium on development. Proc Natl Acad Sci U S A 93: 8455-8459.
29. Stachel SE, Grunwald DJ, Myers PZ (1993) Lithium perturbation and goosecoid expression identify a dorsal specification pathway in the pregastrula zebrafish. Development 117: 1261-1274.

30. Fekany K, Yamanaka Y, Leung T, Sirotkin HI, Topczewski J, et al. (1999) The zebrafish bozozok locus encodes Dharma, a homeodomain protein essential for induction of gastrula organizer and dorsoanterior embryonic structures. Development 126: 1427-1438.

31. Sirotkin HI, Dougan ST, Schier AF, Talbot WS (2000) bozozok and squint act in parallel to specify dorsal mesoderm and anterior neuroectoderm in zebrafish. Development 127: 2583-2592.

32. Joore J, Fasciana C, Speksnijder JE, Kruijer W, Destree OH, et al. (1996) Regulation of the zebrafish goosecoid promoter by mesoderm inducing factors and Xwnt1. Mech Dev 55: 3-18.

33. Kishimoto Y, Lee KH, Zon L, Hammerschmidt M, Schulte-Merker S (1997) The molecular nature of zebrafish swirl: BMP2 function is essential during early dorsoventral patterning. Development 124: 4457-4466.

34. Scharf SR, Rowning B, Wu M, Gerhart JC (1989) Hyperdorsoanterior embryos from Xenopus eggs treated with D2O. Dev Biol 134: 175-188.

35. Kao KR, Elinson RP (1988) The entire mesodermal mantle behaves as Spemann's organizer in dorsoanterior enhanced Xenopus laevis embryos. Dev Biol 127: 64-77.

36. Kao KR, Masui Y, Elinson RP (1986) Lithium-induced respecification of pattern in Xenopus laevis embryos. Nature 322: 371-373.

37. Kao KR, Elinson RP (1989) Dorsalization of mesoderm induction by lithium. Dev Biol 132: 81-90.

38. Aanstad P, Whitaker M (1999) Predictability of dorso-ventral asymmetry in the cleavage stage zebrafish embryo: an analysis using lithium sensitivity as a dorsoventral marker. Mech Dev 88: 33-41.

39. Stambolic V, Ruel L, Woodgett JR (1996) Lithium inhibits glycogen synthase kinase- 3 activity and mimics wingless signalling in intact cells. Curr Biol 6: $1664-1668$.

40. Buee L, Bussiere T, Buee-Scherrer V, Delacourte A, Hof PR (2000) Tau protein isoforms, phosphorylation and role in neurodegenerative disorders. Brain Res Brain Res Rev 33: 95-130.

41. Johnson GV, Hartigan JA (1999) Tau protein in normal and Alzheimer's disease brain: an update. J Alzheimers Dis 1: $329-351$.

42. Dorsky RI, Sheldahl LC, Moon RT (2002) A transgenic Lefl/beta-catenindependent reporter is expressed in spatially restricted domains throughout zebrafish development. Dev Biol 241: 229-237.

43. Taelman VF, Dobrowolski R, Plouhinec JL, Fuentealba LC, Vorwald PP, et al. (2010) Wnt signaling requires sequestration of glycogen synthase kinase 3 inside multivesicular endosomes. Cell 143: 1136-1148.

44. Sconzo G, Cascino D, Amore G, Geraci F, Giudice G (1998) Effect of the IMPase inhibitor L690,330 on sea urchin development. Cell Biol Int 22: 91-94.

45. Westfall TA, Hjertos B, Slusarski DC (2003) Requirement for intracellular calcium modulation in zebrafish dorsal-ventral patterning. Dev Biol 259: 380-391.

46. Westerfield M (1993) The zebrafish book: a guide for the laboratory use of zebrafish (Brachydanio rerio). Eugene, OR: M. Westerfield. 1 v. (unpaged) p.

47. Umbhauer M, Djiane A, Goisset C, Penzo-Mendez A, Riou JF, et al. (2000) The C-terminal cytoplasmic Lys-thr-X-X-X-Trp motif in frizzled receptors mediates Wnt/beta-catenin signalling. EMBO J 19: 4944-4954.

48. Inoue D, Wittbrodt J (2011) One for all - a highly efficient and versatile method for fluorescent immunostaining in fish embryos. PLoS One 6: e19713. 